\title{
Temporary biventricular pacing decreases the vasoactive-inotropic score after cardiac surgery: A substudy of a randomized clinical trial
}

\author{
Huy V. Nguyen, BA, ${ }^{a}$ Vinod Havalad, MD, ${ }^{\mathrm{b}}$ Linda Aponte-Patel, MD, ${ }^{\mathrm{b}}$ Alexandra Y. Murata, BA, ${ }^{\mathrm{c}}$ \\ Daniel Y. Wang, MD, ${ }^{\mathrm{d}}$ Alexander Rusanov, MD, ${ }^{\mathrm{e}}$ Bin Cheng, PhD, ${ }^{\mathrm{f}}$ Santos E. Cabreriza, MBA, ${ }^{\mathrm{c}}$ and \\ Henry M. Spotnitz, $\mathrm{MD}^{\mathrm{c}}$
}

\begin{abstract}
Objective: Vasoactive medications improve hemodynamics after cardiac surgery but are associated with high metabolic and arrhythmic burdens. The vasoactive-inotropic score was developed to quantify vasoactive and inotropic support after cardiac surgery in pediatric patients but may be useful in adults as well. Accordingly, we examined the time course of this score in a substudy of the Biventricular Pacing After Cardiac Surgery trial. We hypothesized that the score would be lower in patients randomized to biventricular pacing.
\end{abstract}

\begin{abstract}
Methods: Fifty patients selected for increased risk of left ventricular dysfunction after cardiac surgery and randomized to temporary biventricular pacing or standard of care (no pacing) after cardiopulmonary bypass were studied in a clinical trial between April 2007 and June 2011. Vasoactive agents were assessed after cardiopulmonary bypass, after sternal closure, and 0 to 7 hours after admission to the intensive care unit.
\end{abstract}

Results: Over the initial 3 collection points after cardiopulmonary bypass (mean duration, 131 minutes), the mean vasoactive-inotropic score decreased in the biventricular pacing group from $12.0 \pm 1.5$ to $10.5 \pm 2.0$ and increased in the standard of care group from $12.5 \pm 1.9$ to $15.5 \pm 2.9$. By using a linear mixed-effects model, the slopes of the time courses were significantly different $(P=.02)$ and remained so for the first hour in the intensive care unit. However, the difference was no longer significant beyond this point $(P=.26)$.

Conclusions: The vasoactive-inotropic score decreases in patients undergoing temporary biventricular pacing in the early postoperative period. Future studies are required to assess the impact of this effect on arrhythmogenesis, morbidity, mortality, and hospital costs. (J Thorac Cardiovasc Surg 2013;146:296-301)

The inotropic score was introduced by Wernovsky and colleagues ${ }^{1}$ as a quantitative measure of cardiovascular support received by neonates after the arterial switch operation. The score has since been used to assess illness severity in infants and neonates undergoing cardiothoracic surgery. ${ }^{2-8}$ Gaies and colleagues ${ }^{9}$ recently introduced the vasoactiveinotropic score (VIS), which expanded on the inotrope score to include other vasoactive agents commonly used in intensive care units (ICUs). The authors showed that the VIS was superior to the older inotrope score as a surrogate outcome measure for infants undergoing cardiac surgery. However, the VIS has not been evaluated in the adult population as a measure of cardiovascular support or severity of illness after cardiopulmonary bypass (CPB).

From the Columbia University College of Physicians and Surgeons, ${ }^{a}$ New York, NY; and the Departments of Pediatrics, ${ }^{\mathrm{b}}$ Surgery, ${ }^{\mathrm{c}}$ Medicine, ${ }^{\mathrm{d}}$ Anesthesiology, ${ }^{\mathrm{e}}$ and Biostatistics, ${ }^{f}$ Mailman School of Public Health, Columbia University, New York, NY.

Funding: A grant from the National Institutes of Health (RO1 HL080152 to H.M.S.). Dr Spotnitz is the George H. Humphreys, II, Professor of Surgery. Mr Nguyen is supported by funds provided to Columbia University by the Weinstein Foundation. Disclosures: Authors have nothing to disclose with regard to commercial support.

Received for publication March 30, 2012; revisions received June 5, 2012; accepted for publication July 10, 2012; available ahead of print July 30, 2012.

Address for reprints: Henry M. Spotnitz, MD, Department of Surgery, Columbia University Medical Center, 622 West 168th St, 10th Floor, Vanderbilt Clinic, Rm 1010,

New York, NY 10032 (E-mail: hms2@columbia.edu).

0022-5223/\$36.00

Copyright (c) 2013 by The American Association for Thoracic Surgery

http://dx.doi.org/10.1016/j.jtcvs.2012.07.020
Vasoactive and inotropic medications currently are useful for the treatment of hypotension and low output states after cardiac surgery. These agents may improve cardiac output but at the significant cost of increases in myocardial oxygen consumption (MVO2), arrhythmias, systemic hypoperfusion, and risk of myocardial ischemia and necrosis. ${ }^{10-13}$

Biventricular pacing (BiVP) is an established therapy for select patients with chronic congestive heart failure, improving clinical end points (symptoms, exercise capacity, quality of life, survival) and echocardiographic end points (systolic function, left ventricular size, mitral regurgitation). ${ }^{14-17}$ Temporary BiVP also has been shown to improve intraoperative cardiac output in patients undergoing cardiac surgery. ${ }^{18-22}$ One unexplored benefit of BiVP is its potential to decrease the use of inotropes and vasoactive medications after cardiac surgery. BiVP does not increase $\mathrm{MVO} 2$ and thus may provide a safer alternative to inotropic medications. ${ }^{11}$

The Biventricular Pacing After Cardiac Surgery (BiPACS) trial is a randomized clinical trial studying the effect of optimized temporary BiVP in selected patients undergoing cardiac surgery. ${ }^{18-21}$ Patients undergo BiVP optimization at 3 time points in the postoperative period and are randomized to continuous optimized BiVP or standard of care (SOC) (no pacing). The central hypothesis is that the cardiac index will increase an average of $15 \%$ in the optimized BiVP group. 


$$
\begin{aligned}
& \text { Abbreviations and Acronyms } \\
& \qquad \begin{array}{ll}
\text { BiPACS } & \text { Biventricular Pacing After Cardiac } \\
& \text { Surgery } \\
\text { BiVP } & =\text { biventricular pacing } \\
\mathrm{CPB} & =\text { cardiopulmonary bypass } \\
\mathrm{ICU} & =\text { intensive care unit } \\
\mathrm{MVO} 2 & =\text { myocardial oxygen consumption } \\
\text { SOC } & =\text { standard of care } \\
\text { VIS } & =\text { vasoactive-inotropic score } \\
\mathrm{VIS}_{\max } & =\text { maximum vasoactive-inotropic score }
\end{array}
\end{aligned}
$$

In this BiPACS substudy, we hypothesized that temporary BiVP would lower the perioperative requirements for vasoactive medication support, as measured by the VIS. Although temporary BiVP has been shown to improve postoperative hemodynamics, there have been no studies investigating whether it reduces the need to administer perioperative inotropic agents.

\section{MATERIALS AND METHODS Study Design}

The BiPACS protocol was approved by the Institutional Review Board for Human Subject Research at Columbia University Medical Center and conducted under an Investigational Device Exemption from the Food and Drug Administration. Attending surgeon permission was obtained before approaching patients for enrollment. Written consent was obtained from all patients.

This unblinded observational study was conducted using a subgroup analysis for eligible patients enrolled in the BiPACS trial between October 1, 2007, and June 30, 2011. Patient flow for the BiPACS trial and this substudy cover recruitment during this time period. A total of 5101 patients underwent cardiac surgery at Columbia University Medical Center and were screened for the BiPACS trial. Of these patients, 764 were eligible for the BiPACS protocol; 105 patients were enrolled. The study was initiated in 57 patients, who were allocated to testing in phase I and subsequently randomized to BiVP (experimental group) or SOC (control group) as per the BiPACS protocol.

Patients were randomized after phase I testing confirmed that BiVP was feasible. To avoid imbalances using simple randomization, patients were randomized to the 2 arms using randomly permuted blocks of 4,6 , and 8. A treatment allocation ratio of 1 was used, expecting each group to be of equal size. Phase 1 testing was done in all patients before randomization and has been described. ${ }^{21}$ Group assignment was determined by forms in sealed envelopes opened at the randomization point. All required forms were prepared before the enrollment of the first patient. Seven patients were eliminated from this substudy because they were removed from the BiPACS trial before the start of phase III. In the end, 24 patients randomized to the BiVP group and 26 patients randomized to the SOC group were available and included in this substudy. The baseline clinical characteristics of these 50 patients are shown in Table 1 .

\section{Biventricular Pacing After Cardiac Surgery Optimization Protocol}

The BIPACS trial is delineated by optimizations at 3 distinct time points ${ }^{21}$ to determine the optimal pacing site, atrioventricular delay, and interventricular delay (Figure 1). Phase I optimization is performed after the patient is separated from CPB and deemed hemodynamically stable by the clinical team. Phase II optimization is performed during sternal closure at the end of the surgery. Finally, phase III optimization is performed 8 to 24
TABLE 1. Baseline characteristics

\begin{tabular}{lccc}
\hline \multicolumn{1}{c}{ Characteristic } & $\begin{array}{c}\text { Randomized } \\
\text { to BiVP } \\
(\mathbf{n}=\mathbf{2 4})\end{array}$ & $\begin{array}{c}\text { Randomized } \\
\text { to SOC } \\
(\mathbf{n}=\mathbf{2 6})\end{array}$ & $\begin{array}{c}\boldsymbol{P} \\
\text { value }\end{array}$ \\
\hline Mean age, y (range) & $69.0(49-85)$ & $66.8(48-87)$ & .46 \\
Female, \% & $17 \%$ & $23 \%$ & \\
Median BMI, $\mathrm{kg} / \mathrm{m}^{2}$ (range) & $25.3(19-46)$ & $26.4(16-41)$ & .77 \\
Mean weight (kg) & $78.5(48-123)$ & $76.4(50-104)$ & .66 \\
Mean CPB time (min) & $133.0(57-325)$ & $132.5(68-239)$ & .83 \\
Mean crossclamp time (min) & $90.9(0-235)$ & $77.8(0-210)$ & .35 \\
Type of surgery* & & & \\
$\quad$ CABG (32) & 13 & 19 & .17 \\
Aortic valve replacement (27) & 15 & 12 & .26 \\
Mitral valve repair or & 11 & 11 & .61 \\
$\quad$ replacement (22) & & & \\
\hline BiVP, Bivin
\end{tabular}

$B i V P$, Biventricular pacing; $S O C$, standard of care (no pacing); $B M I$, body mass in$\operatorname{dex} ; C P B$, cardiopulmonary bypass; $C A B G$, coronary artery bypass grafting. *Several patients underwent combined surgery.

hours after phase II optimization. Patients are randomized to the optimized BiVP group or the SOC group at the end of phase I optimization. Phase I optimization is performed using cardiac output by aortic flow probe, phase II is performed using mean arterial pressure, and phase III optimization is performed using a combination of mean arterial pressure and cardiac output by thermodilution. During periods of BiVP optimization ( 8 minutes during phase I and 16 minutes during phase II), the rates of administration of fluids, blood products, anesthetics, and vasoactive medications are held constant, except for windows for adjustment between optimization of individual parameters.

\section{Anesthesia Protocol}

General endotracheal anesthesia is used for all patients, and dosing is at the discretion of the attending anesthesiologist. Fentanyl and midazolam are used as adjunct agents to attenuate autonomic responses. Inotropes, vasopressors, and vasodilators are administered at the discretion of the attending anesthesiologist on the basis of hemodynamics and real-time transesophageal echocardiography. In general, norepinephrine infusion is the first-line vasoconstrictor, and vasopressin infusion is added if additional vasoconstriction is desirable. The primary inotropic agent is milrinone. The majority of patients undergoing coronary artery bypass grafting with the use of bilateral internal thoracic arteries receive a low-dose $(5 \mu \mathrm{g} / \mathrm{min}) \mathrm{ni}-$ troglycerin infusion at the conclusion of CPB. This infusion is maintained for at least 24 hours postoperatively.

\section{Vasoactive-Inotropic Score}

We used a modification of the VIS described by Gaies and colleagues. ${ }^{9}$ The score at each time point was based on the concurrent doses and types of inotropic and vasopressor medication being administered. We expanded this formula to include the vasopressor phenylephrine and chose its coefficient in the same manner as originally described by Wernovsky and colleagues. ${ }^{1}$ The VIS is calculated as follows:

$$
\begin{aligned}
\text { VIS }= & \text { dopamine dose }(\mu g / K g / \min )+\text { dobutamine dose }(\mu g / K g / \mathrm{min}) \\
& +100 \times \text { epinephrine dose }(\mu g / K g / \mathrm{min}) \\
& +10 \times \text { milrinone dose }(\mu g / K g / \mathrm{min}) \\
& +10,000 \times \text { vasopressin } \operatorname{dose}(U / K g / \mathrm{min}) \\
& +100 \times \text { norepinephrine } \operatorname{dose}(\mu g / K g / \mathrm{min}) \\
& +10 \times \text { phenylephrine } \operatorname{dose}(\mu g / K g / \mathrm{min}) .
\end{aligned}
$$

Specific vasoactive agents used are presented in Table 2. We normalized dosages for patient weight as described previously. $2,7,8$ 


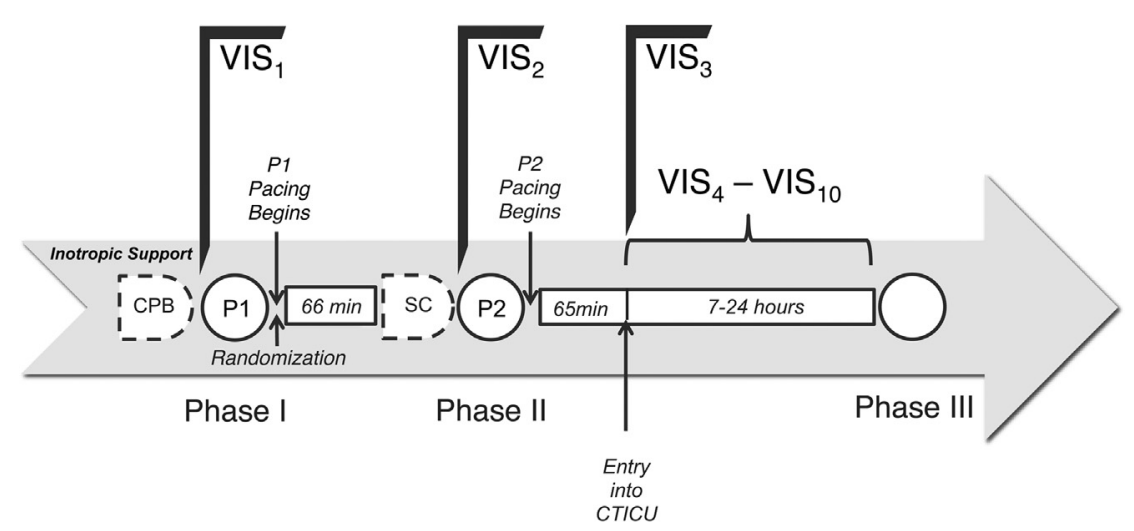

FIGURE 1. Time course of BiPACS protocol. During phase I, after weaning from CPB, the protocol maximizing cardiac output was determined. This protocol was designated P1. Patients were randomized after phase I into the BiVP or SOC arm. Patients in the BiVP arm were paced under P1 until phase II. During phase II, after sternal closure, the protocol maximizing mean arterial pressure was determined and designated $\mathrm{P} 2$. Pacing was then resumed using $\mathrm{P} 2$ in the BiVP arm until phase III optimization, at which point the study period was concluded. VIS was calculated before randomization (VIS $)_{1}$, before phase II $\left(\mathrm{VIS}_{2}\right)$, on relocation from the operating room to the ICU $\left(\mathrm{VIS}_{3}\right)$, and hourly when the patient was in the ICU (VIS 4 -VIS 10 ). VIS, Vasoactiveinotropic score; $C P B$, cardiopulmonary bypass; $S C$, sternal closure; $C T I C U$, cardiothoracic intensive care unit.

VIS $_{1}$ is calculated on all patients immediately preceding phase I optimization. $\mathrm{VIS}_{2}$ is calculated immediately preceding phase II optimization. VIS $_{3}$ is calculated on entry into the ICU. VIS Vo $_{4}$ VIS $_{10}$ are then calculated hourly until the end of the study period (immediately preceding phase III optimization) (Figure 1). The average time between the onset of phase I and the onset of phase II was $66 \pm 30$ minutes, and the average time between the onset of phase II and entry into the ICU was $65 \pm 35$ minutes.

\section{Maximum Vasoactive-Inotropic Score}

The VIS is calculated for each hour the patient spent in the ICU. The maximum VIS (VIS max $_{\text {) }}$ ) is defined as the highest VIS before phase III.

\section{Other Variables}

Urine output produced for each patient after CPB was determined by measuring the volume of urine in the catheter bag on admission to ICU. The patient's serum glucose levels were measured hourly in the ICU.

\section{Statistical Analysis}

The VIS was compared between the BiVP group and the SOC group under linear mixed-effects models where VIS was the outcome and pacing group (BiVP vs SOC) and time (since the start of phase I) were the 2 main predictors. Pacing group by time interaction was also included in the initial model but excluded from the final model if not significant. The analysis also included the subject random effects to account for within subject correlation on the VIS. In addition, the VIS max $_{\max }$ was calculated for each patient after arrival into the ICU and before the start of phase III.

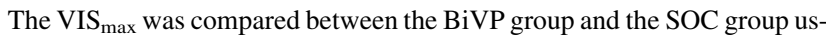
ing an independent 2-sample $t$ test. All the analyses were conducted in SAS version 9.2 (SAS Institute Inc, Cary, NC).

\section{RESULTS}

Figure 2 illustrates the variation of the mean VIS for patients in the temporary BiVP and SOC groups from the end of CPB to admission into the ICU. Both groups enter phase I with a similar VIS, but the scores subsequently diverge, increasing in the SOC group and decreasing in the BiVP group. The difference in mean VIS between the SOC and BiVP groups is statistically significant $(P=.02)$ from the beginning of phase $\mathrm{I}$ to the entry into the ICU (mean duration, 131 minutes). This represents a significant interaction between pacing group and time. There were no significant baseline differences between the 2 groups, as shown in Table 1. In particular, patient weight was the same, which is important, given the per-weight dosing of medications.

TABLE 2. Vasoactive agents used by phase

\begin{tabular}{|c|c|c|c|}
\hline Time & Vasoactive agent & $\begin{array}{c}\text { Randomized } \\
\text { to BiVP } \\
(\mathrm{n}=24)\end{array}$ & $\begin{array}{c}\text { Randomized } \\
\text { to SOC } \\
(\mathrm{n}=\mathbf{2 6})\end{array}$ \\
\hline \multirow[t]{7}{*}{ Entering phase I (OR) } & Dopamine & $0(0 \%)$ & $1(4 \%)$ \\
\hline & Dobutamine & $1(4 \%)$ & $2(8 \%)$ \\
\hline & Epinephrine & $2(8 \%)$ & $3(12 \%)$ \\
\hline & Milrinone & $14(58 \%)$ & $17(65 \%)$ \\
\hline & Norepinephrine & $21(88 \%)$ & $22(85 \%)$ \\
\hline & Vasopressin & $18(75 \%)$ & $21(81 \%)$ \\
\hline & Phenylephrine & $2(8 \%)$ & $1(4 \%)$ \\
\hline \multirow[t]{7}{*}{ Entering phase II (OR) } & Dopamine & $0(0 \%)$ & $1(4 \%)$ \\
\hline & Dobutamine & $1(4 \%)$ & $2(8 \%)$ \\
\hline & Epinephrine & $2(8 \%)$ & $3(12 \%)$ \\
\hline & Milrinone & $14(58 \%)$ & $17(65 \%)$ \\
\hline & Norepinephrine & $21(88 \%)$ & $23(88 \%)$ \\
\hline & Vasopressin & $17(71 \%)$ & $22(85 \%)$ \\
\hline & Phenylephrine & $2(8 \%)$ & $1(4 \%)$ \\
\hline \multirow[t]{7}{*}{ Before phase III (ICU) } & Dopamine & $0(0 \%)$ & $2(8 \%)$ \\
\hline & Dobutamine & $3(13 \%)$ & $2(8 \%)$ \\
\hline & Epinephrine & $3(13 \%)$ & $5(19 \%)$ \\
\hline & Milrinone & $14(58 \%)$ & $17(65 \%)$ \\
\hline & Norepinephrine & $22(92 \%)$ & $22(85 \%)$ \\
\hline & Vasopressin & $19(79 \%)$ & $23(88 \%)$ \\
\hline & Phenylephrine & $1(4 \%)$ & $3(12 \%)$ \\
\hline
\end{tabular}

BiVP, Biventricular pacing; $S O C$, standard of care (no pacing); $O R$, operating room; $I C U$, intensive care unit. 


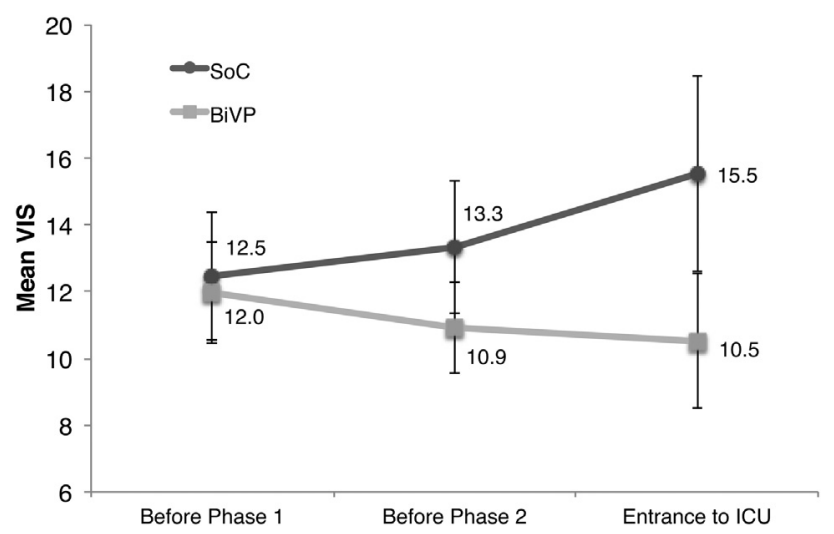

FIGURE 2. Diverging time course from randomization to ICU admission in the SOC $(\mathrm{n}=26)$ and $\operatorname{BiVP}(\mathrm{n}=24)$ groups. The VIS increased from $12.5 \pm 1.9$ at randomization to $15.5 \pm 2.9$ at ICU entry in the SOC group but decreased from $12.0 \pm 1.5$ to $10.5 \pm 2.0$ in the BiVP group. The slopes of these VIS-time relations are significantly different by linear mixedeffects analysis $(P=.02)$. The time averaged $66 \pm 30$ minutes between phase I and II and $65 \pm 35$ minutes between phase II and ICU entry. Pacing was optimized at time points $\mathrm{VIS}_{1}$ and $\mathrm{VIS}_{2}$. VIS, Vasoactive-inotropic score; $I C U$, intensive care unit; $B i V P$, biventricular pacing; SoC, standard of care.

Figure 3 illustrates the time course of the mean VIS of the 2 groups from the point of randomization to 7 hours after entry into the ICU. Pacing optimization for patients in the BiVP group was not repeated after sternal closure until phase III, which was later than the data in Figure 3. The observation period in the ICU was based on the BiPACS protocol, which ended if the Swan-Ganz catheter (Edwards
LifeSciences, Irvine, Calif) was removed or after 24 hours in the ICU, whichever came first. To standardize the data, the VIS was computed only through the first 7 hours, which all patients had in common. The data were analyzed under a piecewise linear mixed-effects model with the focal time period being 1 hour after entrance into the ICU. The difference in the change of VIS between the BiVP group and SOC, measured each hour, remains significant up to the first hour in the ICU, which extends the therapeutic effect of BiVP as measured by the VIS to a total of 3 hours after randomization (Figure 3, $P=.0015$ ).

Group differences in the VIS after 1 hour in the ICU are no longer statistically significant $(P=.26)$. This $P$ value represents an insignificant difference in pacing group effect, although the time effect was significant (coefficient $=0.28$, $P=.02$ ).

When the mean VIS $_{\text {max }}$ is compared between the 2 groups, there is a trend toward a lower VIS $_{\max }$ in the BiVP group, but the difference is not significant (BiVP mean, $17.6 \pm 2.5$; SOC mean, $22.4 \pm 4.3 ; P=.33$ ).

Urine output from the conclusion of CPB to ICU admission was significantly higher in patients randomized to BiVP than in patients randomized to SOC $(7.0 \pm 1.7 \mathrm{~mL} /$ $\mathrm{h} / \mathrm{kg}$ vs $2.6 \pm 0.5 \mathrm{~mL} / \mathrm{h} / \mathrm{kg}, P=.012)$. The concurrent maximal glucose levels were similar in both groups $(168 \pm 8$ $\mathrm{mg} / \mathrm{dL}$ vs $181 \pm 13 \mathrm{mg} / \mathrm{dL}$, respectively, $P=.42$ ).

\section{DISCUSSION}

Previous data from the BiPACS trial have demonstrated the hemodynamic superiority of temporary BiVP versus

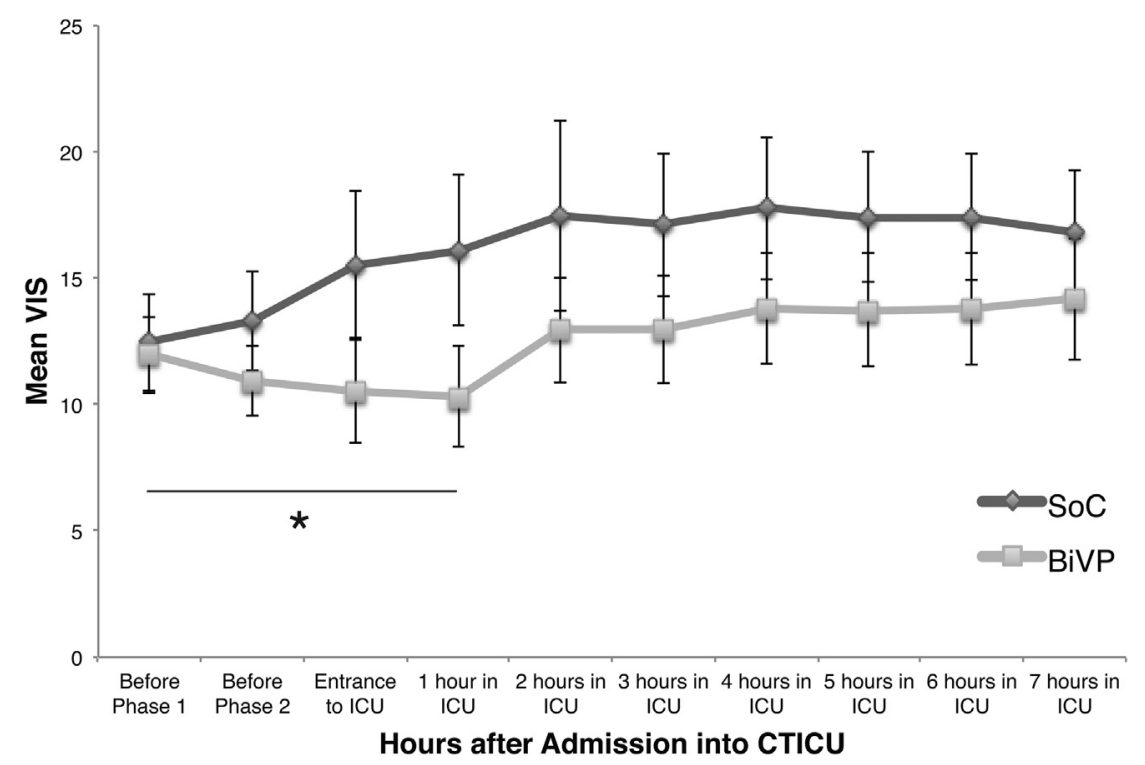

FIGURE 3. VIS from randomization to 7 hours after ICU entry. Hourly averages of the VIS are shown for the SOC $(n=26)$ and BiVP ( $n=24)$ groups. The slopes of the VIS-time relations are significantly different to the first hour in the ICU by linear mixed-effects analysis $(P=.0015)$. Pacing was optimized at time points VIS 1 and VIS 2 . Data collection was limited to 7 hours after ICU entry because earliest Swan-Ganz catheter (Edwards LifeSciences, Irvine, Calif) removal occurred at this time. VIS, Vasoactive-inotropic score; $I C U$, intensive care unit; $C T I C U$, cardiothoracic intensive care unit; $B i V P$, biventricular pacing; $S o C$, standard of care. 
SOC, as judged by increases in cardiac output or mean arterial pressure. ${ }^{21,23}$ However, changes in inotrope and vasoactive agent use associated with temporary BiVP were not examined.

The present results demonstrate decreasing VIS in the temporary BiVP group versus the SOC group over time, suggesting that superior hemodynamics resulted in the decreased administration of inotropes and vasoactive agents. We have not defined a mechanism for the implied feedback of hemodynamics on the rate of infusion of vasoactive drugs, and use of these agents is not regulated by protocol at Columbia University Medical Center. Because weaning of vasoactive drugs is generally based on the time course of arterial pressure, urine output, and cardiac output, we speculate that differences in VIS between our patient groups reflect the patient care team's perception of changes in these parameters.

Although several previous studies have demonstrated hemodynamic benefits related to temporary BiVP, demonstration of benefits in outcome has been elusive. However, Gaies and colleagues ${ }^{9}$ reported that $\mathrm{VIS}_{\max }$ in the ICU can predict morbidity and mortality; thus, the present results suggest that such benefits might be demonstrable in a larger study. Inotropes and vasopressors in the perioperative period can support cardiac output, regulate myocardial contractility, improve hemodynamics, and reduce left and right ventricular filling pressures. ${ }^{10}$ However, inotropes are proarrhythmic and increase MVO2. The latter can induce myocardial ischemia and adversely affect reperfusion injury after $\mathrm{CPB} .{ }^{10-13}$ Under well-controlled conditions, BiVP can increase ventricular function without increasing $\mathrm{MVO} 2,{ }^{11}$ an appealing aspect of this therapy.

The present results demonstrate that adults who receive optimized temporary BiVP require lower levels of vasoactive and inotropic support during the early postoperative period (Figures 2 and 3). This could reflect increased cardiac output in patients receiving temporary BiVP versus SOC. Because inotropes are linked to arrhythmogenesis, reduced use during the early postoperative period could decrease the patient's risk of a complicating arrhythmia. The reduced use of inotropes is further beneficial in reducing $\mathrm{MVO} 2$ and thus decreasing the chance of reperfusion injury after $\mathrm{CPB}$.

Our results suggest that the benefit of temporary BiVP pacing begins immediately and remains significant as long as the pacing protocol is optimized hourly. We find a statistically significant reduction in vasoactive and inotropic support in the BiVP group for 2 hours after the last optimization of pacing. This suggests that deterioration of the effects of temporary BiVP may be due to the lack of continued pacing optimization, and further indicates that effects of temporary BiVP would be maximized by continuous optimization during the postoperative period.

In addition, a significant increase in urine output was observed for the BiVP group versus the SOC group from the beginning of phase I to entry into the ICU. Although this study was not designed for a multifactorial assessment of factors affecting urine output, this result, which mirrored our VIS analysis, further supports the postoperative cardiovascular benefit of optimized temporary BiVP.

The VIS was validated in pediatric patients undergoing cardiac surgery as a marker of inotropic support and an established predictor of outcome. ${ }^{9,24}$ However, until now, there have been no studies using this updated vasoactive inotrope score in the adult population. The VIS in adults is expected to differ from the VIS in infants because the response of cardiac muscle and vasculature to inotropic and vasopressor mediations differs between pediatric and adult patients. ${ }^{25}$ The VIS clearly merits further evaluation as an adjunct to management of adult patients after CPB. The present results also support the value of temporary BiVP as an inotrope replacement. Further studies are needed of both the VIS in adults and the hemodynamics of temporary BiVP after CPB.

It is important to note that the VIS focuses on the amount, not the specific types, of vasoactive and inotropic support. Specific agents are used at the individual clinician's discretion to improve outcome and severity of illness. This nonstandardized protocol cannot be controlled for in a retrospective study such as ours, so prospective randomized trials are necessary to account for selection bias and optimize the strategy for implementing specific medications.

\section{Study Limitations}

There are several additional limitations to our study. This study is part of the larger BiPACS trial. An independent justification of sample size for this substudy would be based on anticipated differences in the VIS between the 2 groups and on the variance of the VIS. This would define an ideal sample size larger than presented in the current study. Termination of the parent trial precludes additional data collection. However, statistical significance was achieved by fitting linear effects models over time and comparing the slope. Anesthesiologists were not blinded to the 2 arms of the study; however, they were also not aware that the VIS would be evaluated. In addition, this is a single-center study, and thus our findings may not be generalizable to other patient populations.

\section{CONCLUSIONS}

Our results show that optimized temporary BiVP after CPB lowers the requirement for inotropic support in the early postoperative period, therefore decreasing the exposure to inotrope-associated risks. The VIS can be useful in post-CPB management and pacing intervention as a tool to quantify vasoactive and inotropic support, as well as a potential measure of cardiovascular function. The VIS has the potential to be a component of a multivariable severity of illness scoring system, which would be particularly useful to practitioners trying to decide between various diagnostic 
and therapeutic options for patients recovering from cardiac surgery with BiVP. Additional studies are required to show a correlation between VIS and outcomes, such as arrhythmias, morbidity, or mortality.

\section{References}

1. Wernovsky G, Wypij D, Jonas RA, Mayer JE, Hanley FL, Hickey PR, et al. Postoperative course and hemodynamic profile after the arterial switch operation in neonates and infants. A comparison of low-flow cardiopulmonary bypass and circulatory arrest. Circulation. 1995;92:2226-35.

2. Talwar S, Sandeep JA, Choudhary SK, Velayoudham D, Lakshmy R, Kasthuri JM, et al. Effect of preoperative administration of allopurinol in patients undergoing surgery for valvular heart diseases. Eur J Cardiothorac Surg. 2010; 38:86-90.

3. Rhodes JF, Blaufox AD, Seiden HS, Asnes JD, Gross RP, Rhodes JP, et al. Cardiac arrest in infants after congenital heart surgery. Circulation. 1999;100(19 Suppl):II194-9.

4. Gruenwald CE, McCrindle BW, Crawford-Lean L, Holtby H, Parshuram C, Massicotte P, et al. Reconstituted fresh whole blood improves clinical outcomes compared with stored component blood therapy for neonates undergoing cardiopulmonary bypass for cardiac surgery: a randomized controlled trial. J Thorac Cardiovasc Surg. 2008;136:1442-9.

5. Kulik TJ, Moler FW, Palmisano JM, Custer JR, Mosca RS, Bove EL, et al. Outcome-associated factors in pediatric patients treated with extracorporeal membrane oxygenator after cardiac surgery. Circulation. 1996;94(9 Suppl):II63-8.

6. Rosenzweig EB, Starc TJ, Chen JM, Cullinane S, Timchak DM, Gersony WM, et al. Intravenous arginine-vasopressin in children with vasodilatory shock after cardiac surgery. Circulation. 1999;100(19 Suppl):II182-6.

7. Yang L, Yang J, Wang Q, Chen M, Lu Z, Chen S, et al. Cardioprotective effects of electroacupuncture pretreatment on patients undergoing heart valve replacement surgery: a randomized controlled trial. Ann Thorac Surg. 2010;89:781-6.

8. Ricci Z, Morelli S, Ronco C, Polito A, Stazi GV, Giorni C, et al. Inotropic support and peritoneal dialysis adequacy in neonates after cardiac surgery. Interact Cardiovasc Thorac Surg. 2008;7:116-20.

9. Gaies MG, Gurney JG, Yen AH, Napoli ML, Gajarski RJ, Ohye RG, et al. Vasoactive-inotropic score as a predictor of morbidity and mortality in infants after cardiopulmonary bypass. Pediatr Crit Care Med. 2010;11:234-8.

10. Parissis JT, Rafouli-Stergiou P, Stasinos V, Psarogiannakopoulos P, Mebazaa A. Inotropes in cardiac patients: update 2011. Curr Opin Crit Care. 2010;16:432-41.

11. Nelson GS, Berger RD, Fetics BJ, Talbot M, Spinelli JC, Hare JM, et al. Left ventricular or biventricular pacing improves cardiac function at diminished energy cost in patients with dilated cardiomyopathy and left bundle-branch block. Circulation. 2000;102:3053-9.
12. Lewis KP. Early intervention of inotropic support in facilitating weaning from cardiopulmonary bypass: the New England Deaconess Hospital experience. $J$ Cardiothorac Vasc Anesth. 1993; 7(4 Suppl 2):40-5.

13. Cuffe MS, Califf RM, Adams KF Jr, Benza R, Bourge R, Colucci WS, et al Short-term intravenous milrinone for acute exacerbation of chronic heart failure: a randomized controlled trial. JAMA. 2002;287:1541-7.

14. Auricchio A, Stellbrink C, Sack S, Block M, Vogt J, Bakker P, et al. Long-term clinical effect of hemodynamically optimized cardiac resynchronization therapy in patients with heart failure and ventricular conduction delay. J Am Coll Cardiol. 2002;39:2026-33.

15. Abraham WT, Fisher WG, Smith AL, Delurgio DB, Leon AR, Loh E, et al. Cardiac resynchronization in chronic heart failure. N Engl J Med. 2002;346: 1845-53.

16. Bax JJ, Abraham T, Barold SS, Breithardt OA, Fung JW, Garrigue S, et al. Cardiac resynchronization therapy: part 1 -issues before device implantation. $J \mathrm{Am}$ Coll Cardiol. 2005;46:2153-67.

17. Bax JJ, Abraham T, Barold SS, Breithardt OA, Fung JW, Garrigue S, et al. Cardiac resynchronization therapy: part 2 -issues during and after device implantation and unresolved questions. J Am Coll Cardiol. 2005;46:2168-82.

18. Spotnitz ME, Wang DY, Quinn TA, Cheng B, Richmond ME, Rusanov A, et al. Hemodynamic stability during biventricular pacing after cardiopulmonary bypass. J Cardiothorac Vasc Anesth. 2011;25:238-42.

19. Berberian G, Quinn TA, Kanter JP, Curtis LJ, Cabreriza SE, Phillips AB, et al Optimized biventricular pacing in atrioventricular block after cardiac surgery. Ann Thorac Surg. 2005;80:870-5.

20. Quinn TA, Berberian G, Cabreriza SE, Maskin LJ, Weinberg AD, Holmes JW, et al. Effects of sequential biventricular pacing during acute right ventricular pressure overload. Am J Physiol Heart Circ Physiol. 2006;291:H2380-7.

21. Wang DY, Richmond ME, Quinn TA, Mirani AJ, Rusanov A, Yalamanchi V, et al Optimized temporary biventricular pacing acutely improves intraoperative cardiac output after weaning from cardiopulmonary bypass: a substudy of a randomized clinical trial. J Thorac Cardiovasc Surg. 2011;141:1002-8.e1.

22. Weisse U, Isgro F, Werling C, Lehmann A, Saggau W. Impact of atriobiventricular pacing to poor left-ventricular function after CABG. J Thorac Cardiovasc Surg. 2002;50:131-5.

23. Rubinstein B, Wang DY, Cabreriza SE, Richmond ME, Rusanov A, Quinn TA et al. Response of mean arterial pressure to temporary biventricular pacing after chest closure during cardiac surgery. A substudy of the Biventricular Pacing After Cardiac Surgery (BiPACS) trial. J Thorac Cardiovasc Surg. 2012;144:1445-52.

24. Zahorec M, Hrubsova Z, Skrak P, Poruban R, Mosal M, Kovacikova L, et al. A comparison of Blalock-Taussig shunts with and without closure of the ductus arteriosus in neonates with pulmonary atresia. Ann Thorac Surg. 2011;92: 653-8.

25. Rao V, Ivanov J, Weisel RD, Ikonomidis JS, Christakis GT, David TE. Predictors of low cardiac output syndrome after coronary artery bypass. J Thorac Cardiovasc Surg. 1996;112:38-51 